\title{
Nonlocal nonlinear magneto-optical response of a magnetoplasmonic crystal
}

\author{
I. Razdolski, ${ }^{1,}{ }^{*}$ D. G. Gheorghe, ${ }^{1}$ E. Melander, ${ }^{2}$ B. Hjörvarsson, ${ }^{2}$ P. Patoka, ${ }^{3}$ A. V. Kimel, ${ }^{1}$ A. Kirilyuk, ${ }^{1}$ \\ E. Th. Papaioannou, ${ }^{2, \dagger}$ and Th. Rasing ${ }^{1}$ \\ ${ }^{1}$ Radboud University Nijmegen, Institute for Molecules and Materials, 6525 AJ Nijmegen, The Netherlands \\ ${ }^{2}$ Department of Physics and Astronomy, Uppsala University, Box 516, SE-751 20 Uppsala, Sweden \\ ${ }^{3}$ Physikalische und Theoretische Chemie, Institut für Chemie und Biochemie, Freie Universität Berlin, Takustraße 3, 14195 Berlin, Germany
}

(Received 26 March 2013; published 29 August 2013)

\begin{abstract}
Surface plasma resonance (SPR) excitation in a Co-based two-dimensional magnetoplasmonic crystal was found to strongly enhance the second-harmonic generation (SHG) efficiency. Large changes in the phase shift between magnetic and nonmagnetic SHG contributions were observed in the transversal Kerr effect as a function of the incidence angle. The activation of a nonlocal quadrupole mechanism of the magnetic SHG due to the SPR excitation was found to lead to an unusual phase behavior in the vicinity of the resonance.
\end{abstract}

DOI: 10.1103/PhysRevB.88.075436

PACS number(s): 73.20.Mf, 78.20.Ls, 78.66.-w

\section{INTRODUCTION}

Surface plasma resonances (SPRs) in metallic nanostructures provide effective means to localize light in volumes much smaller than the wavelength of the radiation. ${ }^{1-8}$ The intriguing opportunity to integrate photonics and electronics on the nanoscale using such SPRs has triggered a plethora of experimental and theoretical investigations of both linear and nonlinear optical phenomena in plasmonic nanostructures. ${ }^{9-16}$ The latter, though relying on relatively small nonlinear-optical susceptibilities, are significantly enhanced by surface plasmons thus effectively expanding the field of nonlinear-optical applications, including light frequency conversion ${ }^{2}$ and ultrafast switching. ${ }^{17}$ The emerging field of magnetoplasmonics gives a new twist to this appealing research direction as it may open up an effective way to control the frequency converted light with the help of a magnetic field. Indeed, a manyfold enhancement of magneto-optical effects in plasmonic nanostructures has been successfully demonstrated. ${ }^{18-23}$

The conventional approach to treat light-matter interactions is based on the electric dipole approximation in which an incident light wave induces a polarization in the medium. ${ }^{24}$ This polarization is considered as a source for the transmitted and reflected light. In the case of SHG, light at frequency $\omega$ induces an electric polarization at frequency $2 \omega$, which, in turn, emits a second harmonic wave with intensity $I^{2 \omega} \cdot{ }^{25}$ Similarly to linear optics, the nonlinear polarization $\vec{P}^{2 \omega}$ can be a function of the magnetization $\vec{M}$ of the medium. In the lowest order of the expansion with respect to $M$ one can distinguish crystallographic, i.e., magnetization-independent, and magnetic parts of the induced nonlinear polarization: ${ }^{26}$

$$
\vec{P}^{2 \omega}=\hat{\chi}_{c r}^{(2)}: \vec{E}^{\omega} \vec{E}^{\omega}+\hat{\chi}_{\text {mag }}^{(2)}: \vec{E}^{\omega} \vec{E}^{\omega} M(0),
$$

where $\vec{E}^{\omega}$ is the electric field of the fundamental radiation, and $\hat{\chi}_{\text {mag }}^{(2)}$ and $\hat{\chi}_{c r}^{(2)}$ are magnetic and crystallographic susceptibility tensors of a third and second rank, respectively. As such, interference between these two coherent terms determines the total second harmonic $(\mathrm{SH})$ intensity $I^{2 \omega}$, whereas the latter becomes a function of the magnetization. Thus, the magnetic second harmonic generation (MSHG) intensity strongly depends not only on the absolute values of the complex second-order susceptibilities $\left|\chi_{\text {mag }}^{(2)}\right|,\left|\chi_{c r}^{(2)}\right|$ but also on the relative phase $\varphi$ between them: $I^{2 \omega} \approx\left|\chi_{c r}^{(2)}\right|^{2}\left|E^{\omega}\right|^{4}+$ $2\left|\chi_{\text {mag }}^{(2)}\right|\left|\chi_{c r}^{(2)}\right|\left|E^{\omega}\right|^{4} M(0) \cos \varphi$, where the even-in magnetization term $\propto M^{2}(0)$ is neglected as being small compared to the crystallographic one. In a nondissipative approximation $\varphi$ is strictly equal to $\pi / 2 .{ }^{27}$ Even if dissipation is present, if both $\chi_{\mathrm{mag}}^{(2)}$ and $\chi_{c r}^{(2)}$ employ the same optical transition, $\varphi$ is conventionally considered as a wavelength-independent phenomenological parameter. ${ }^{28-31}$

Here we study MSHG in a two-dimensional Co-based magnetoplasmonic crystal as a function of angle of incidence, which determines the relative plasma resonance position with respect to the frequency of light. We show that the phase $\varphi$ between the magnetic and crystallographic contributions in the vicinity of the SPR is extremely sensitive to the angle of incidence. Analyzing this behavior, we demonstrate that the electric dipole approximation fails to describe the observed effects. We show that the changes are due to a nonlocal quadrupole source of MSHG which is activated in the bulk of the Co film, when the SPR is excited.

\section{EXPERIMENT}

The $\mathrm{Au}(2 \mathrm{~nm}) / \mathrm{Co}(60 \mathrm{~nm}) / \mathrm{Ti}(2 \mathrm{~nm}) / \mathrm{Si}(111)$ plasmonic crystal was fabricated by the technology described in Refs. 3234. The $\mathrm{Au}$ and Co layers were decorated with a hexagonal array of circular holes of average diameter of $250 \mathrm{~nm}$ and average pitch size of $470 \mathrm{~nm}$. A continuous film of the same composition served as a reference sample.

Nonlinear-optical measurements were performed on a setup allowing both angular spectra and anisotropic SHG measurements. A tunable (730-900 nm wavelength) Mai-Tai laser with the output power of $100 \mathrm{~mW}$ and pulse duration of $100 \mathrm{fs}$ was used as a fundamental radiation source. The fundamental radiation was always $p$ polarized and was focused onto the samples in a spot of about 50 microns in diameter. An electromagnet was used to saturate the in-plane magnetization of the sample with a field of $100 \mathrm{mT}$.

In Fig. 1, the angular reflectivity spectra of the plasmonic sample are presented for its azimuthal orientation shown in the central inset. The SPR nature of the observed features is clearly discussed in Ref. 34. Empty and full red symbols demonstrate how the SPR (excited along the red arrow, central inset) shifts 


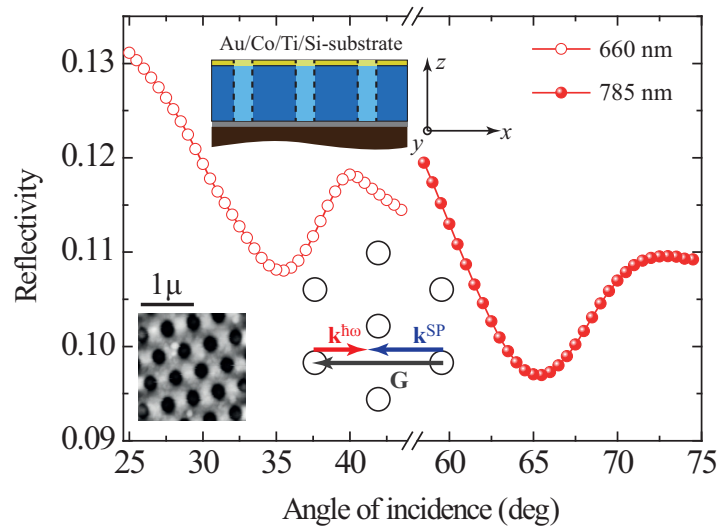

FIG. 1. (Color online) Angular spectra of the linear reflectivity for excitation wavelengths of $660 \mathrm{~nm}$ (empty symbols) and $785 \mathrm{~nm}$ (full symbols). The incident light was polarized in the plane of incidence. Insets: (top) in-depth structure of the perforated sample and the reference frame; (middle) the antidots grid and the $k$ vectors of the excited plasmon ( $\mathbf{k}^{S P}$, blue), light $\left(\mathbf{k}^{\hbar \omega}\right.$, red $)$, and the reciprocal lattice (G, black); (bottom left) an AFM image of a plasmonic sample, showing the hexagonal array of antidots.

with the excitation wavelength. The SPR in the other direction (rotated $30^{\circ}$ ) requires different excitation conditions $\left(\approx 78^{\circ}\right.$ of incidence for the $700 \mathrm{~nm}$ wavelength) and falls outside the range of our setup's tunability, and will not be discussed henceforth.

SHG anisotropy data for the $p$-in, $P$-out polarizations are presented in Fig. 2, where $I^{2 \omega}$ is plotted as a function of azimuthal angle of the sample. The full dots show the anisotropic SHG response in the vicinity of the plasmon resonance (red circles) and far away from it (black diamonds), whereas the empty dots represent the reference plain film data. Angular positions of the maxima correspond to the sample orientations where the proper lattice period is aligned along the electric field of the $p$-polarized fundamental radiation. An SHG signal enhancement by a factor of 6 is clearly observed at the resonance, as compared to the off-resonant conditions. Note that the anisotropy of the SHG response is given not by the $\chi^{(2)}$ tensor, but by the local field factor $L(\omega)$ due to

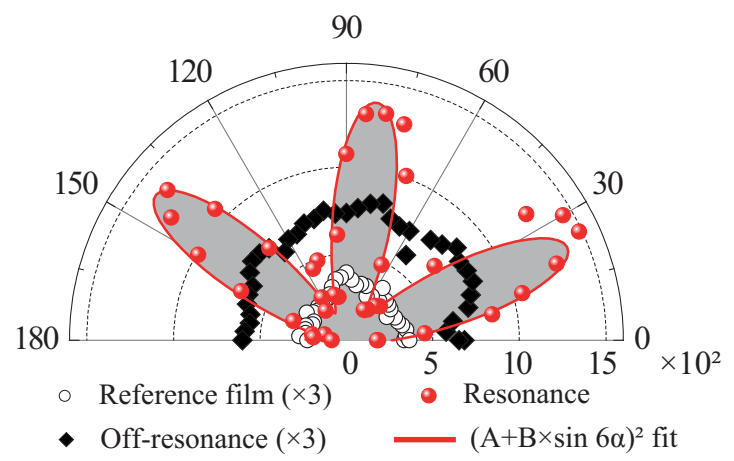

FIG. 2. (Color online) Anisotropic SHG response of the reference sample (empty dots), perforated sample far away (black diamonds), and right at (red dots) the plasmon resonance. The polarization configuration is $p$-in, $P$-out. The symmetry of the SHG intensity is inherited from the SPR excitation conditions resembling the symmetry of the hexagonal array of antidots. the SPR excitation. The symmetry of the SHG signal reflects the sixfold symmetry of the antidots array, indicating the $k$ directions in which the incident field can excite the SPR. Since the SHG response does not exhibit any regular symmetry in the nonresonant case (see empty dots and black diamonds), the buried $\mathrm{Co} / \mathrm{Ti} / \mathrm{Si}(111)$ interfaces appear to hardly give rise to any SHG output.

In order to establish correlations between a SPR and the nonlinear magneto-optical response of the plasmonic crystal, we also performed MSHG measurements. Let us consider a reference frame where $z$ is normal to the sample plane and $x$ lies in the plane of incidence. In the transverse Kerr geometry, when an external magnetic field is directed along the $y$ axis (perpendicular to the plane of incidence), for an isotropic magnetic film in the $p$-in, $P$-out geometry, three even and three odd-in magnetization $\chi^{(2, d)}$ components are excited. These are $\chi_{z z z}, \chi_{z x x}, \chi_{x z x}$ and $\chi_{x z z}, \chi_{x x x}, \chi_{z x z}$, respectively. In this geometry one can expect changes in the MSHG intensity, ${ }^{27}$ conventionally described by means of the magnetic contrast $\rho$ defined as the relative MSHG intensity change upon switching the sample magnetization, $\rho=\left(I_{\uparrow}-I_{\downarrow}\right) /\left(I_{\uparrow}+I_{\downarrow}\right)$. For the reference film, $\rho$ [Fig. 3(a), empty dots] is quite large (80\%) but shows no strong spectral or angular dependence because no SPR is excited in this case. In striking contrast, for the perforated film, the magnetic contrast $\rho$ experiences dramatic changes with angle of incidence [Fig. 3(a), full green dots], including sign reversal. The plasmonic nature of the effect is indicated by the fact that when the fundamental wavelength experiences a redshift, the resonant angle increases according to the SPR excitation relation ${ }^{35} \vec{k}^{S P}=\vec{K}_{0} \pm n \vec{e}_{a} \pm m \vec{e}_{b}$, where $\vec{k}^{S P}$ is the surface plasmon wave vector, the in-plane component of the light wavevector $\vec{k}_{0}$ is $\vec{K}_{0}=(\omega / c)\left(\vec{k}_{0} /\left|\vec{k}_{0}\right|\right) \sin \theta, \omega$ is the frequency, $c$ is the speed of light, $\vec{e}_{a}$ and $\vec{e}_{b}$ are the reciprocal lattice vectors for the hexagonal lattice, $\theta$ is the incident angle, and $n$ and $m$ are integers.

\section{DISCUSSION}

Within the conventional phenomenological magnetooptical model, two main parameters determine the $\rho$ value, namely, the ratio of nonmagnetic to magnetic susceptibility $\left|\chi_{\mathrm{mag}}^{(2)}\right| /\left|\chi_{c r}^{(2)}\right|=\Phi$ and the relative phase $\varphi$ between them. As such, a sign change of the magnetic contrast could stem from a substantial shift of the phase between magnetic and nonmagnetic MSHG contributions. In order to study the phase behavior in the vicinity of the SPR, we performed phase measurements using the nonlinear interferometry method, ${ }^{36-38}$ where the reference quartz sample was translated along the optical beam. The angular dependence of the phase difference $\varphi(\theta)$ extracted from the interference patterns, as described in Ref. 39, is shown in Fig. 3(a) in black diamonds. The angular spectrum demonstrates a significant $90^{\circ}$ phase shift in the vicinity of the SPR.

In order to separate the phases, similar interferometry measurements were performed in the longitudinal Kerr geometry $[M(0)$ is directed along the $x$ axis]. In this case nonmagnetic and magnetic SHG contributions are $p$ and $s$ polarized, respectively, ${ }^{27,40}$ which allows independent studies of each of them. The relative phases of the $p$-polarized (nonmagnetic) 


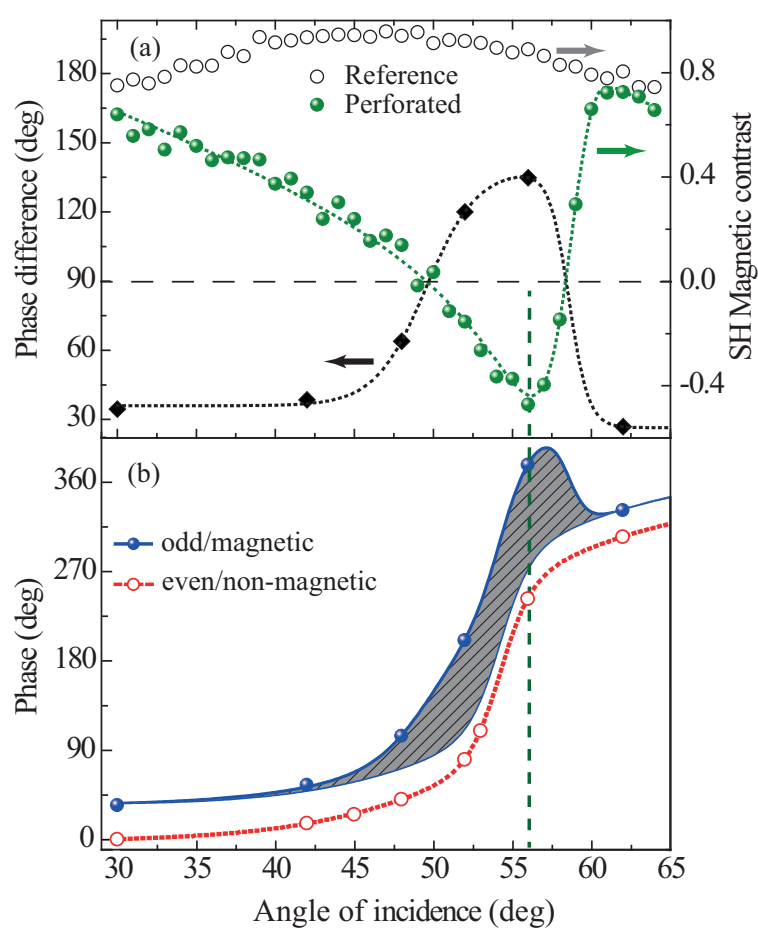

FIG. 3. (Color online) (a) Angular spectra of the SHG magnetic contrast for the reference (empty dots) and perforated (green full dots) films. The polarization configuration is $p$-in, $P$-out, the samples are magnetized in the transverse Kerr geometry, and the fundamental wavelength is $745 \mathrm{~nm}$. Black diamonds represent the angular spectrum of the phase difference between even and odd in-magnetization SHG contributions. (b) Angular spectra of relative phases of the even (red empty dots) and odd (blue full dots) SHG components. The dashed area represents phase shift introduced by the SPR. Dotted lines are the result of an interpolation. The vertical line hints at the SP resonant angle at $745 \mathrm{~nm}$ wavelength.

SHG contribution $\varphi_{N}(\theta)$ were measured in the $p$-in, $P$-out polarization configuration and are presented in Fig. 3(b) with red empty dots. Note that this nonmagnetic SHG contribution is exactly the same in both transversal and longitudinal Kerr geometries. Now it became possible to extract the phase data of the magnetic SHG contribution [Fig. 3(b), full blue dots]. This angular spectrum can be understood as consisting of two different contributions: a generic steplike one with a constant phase shift with respect to the nonmagnetic contribution, and a resonant one [Fig. 3(b), dashed area below the blue line] which exists only in the vicinity of the SPR. The huge magnitude of the effect (about $90^{\circ}$ ) shows that there must be an additional resonant magnetic SHG contribution, with its phase substantially different from the nonresonant one.

The origin of this resonant contribution must be an additional MSHG source, which, within the electric dipole approximation, can be located only at the interfaces, where the inversion symmetry is broken. However, we can exclude the bottom $\mathrm{Co} / \mathrm{Ti} / \mathrm{Si}$ interface due to the following reasons. If the SHG generated at the bottom interface gave any contribution to the observed signal, this would modify both the magnetic and nonmagnetic parts of the SHG response. Also, note that due to the extraordinary transmission induced by the SPR excitation ${ }^{11,41}$ through the perforated Co layer, the sample is relatively transparent for the fundamental beam only, but not for the SHG radiation. This means the latter cannot contribute to the signal if generated too deep. Numerical simulations showed that the relevant transmittivity is only of the order of a few percent for the buried interface-born SHG. As such, we can conclude that the dipole approximation fails to describe the observed phenomena, and the answer may be related to the quadrupole contribution. The latter can be of two origins, namely, intrinsic (microscopic) and nonlocal. However, for the far-field SHG signal they both contribute in a similar way, ${ }^{42}$ although the nonlocal type can be essential in the case of an SPR excitation, as suggested in Ref. 43.

The importance of nonlocal quadrupole SHG sources has been already pointed out in Refs. 16 and 44-46, although an excitation of a nonlocal magnetic SHG has so far not been reported. Plasma resonances efficiently modify the electromagnetic field distribution in the medium, ${ }^{28,47,48}$ which is usually accompanied by large electric field gradients. In our case, the plasmon-enhanced field $E_{z}$ is now not only modulated along the $x$ axis but is also much more strongly concentrated inside the Co layer, as compared with the off-resonant conditions. Thus the plasmon-induced periodic modulation of the $E_{z}$ component can enable an addtitional SHG source in the centrosymmetric Co bulk, which has to be nonlocal due to symmetry reasons.

In general, a nonlocal quadrupole $\mathrm{SHG}$ contribution is described as $\vec{P}(2 \omega, Q)=\chi^{(2)}: \vec{E} \nabla \vec{E}$. Similarly to what has been said about the dipole SHG, magnetic and crystallographic parts of $\vec{P}^{(2 \omega, Q)}$ can be distinguished, ${ }^{26}$ which gives

$$
\vec{P}^{(2 \omega, Q)}=\chi_{c r}^{(2)}: \vec{E}^{\omega} \nabla \vec{E}^{\omega}+\chi_{\text {mag }}^{(2)}: \vec{E}^{\omega} \nabla \vec{E}^{\omega} M(0) .
$$

The field gradient $\nabla \vec{E}^{\omega}$ due to the plasmon wave implies in this case $E_{z} \propto e^{i k x}$, where $k$ is the plasmon wave number. Thus in the $p$-in, $p$-out geometry $P^{(2 \omega, Q)}$ turns into a vector sum of a few terms proportional to $\chi_{\{x, z\}}^{(2)} \vec{e}_{\alpha},{ }^{40}$ the most important of which is $i \vec{e}_{z} \chi_{z x z z}^{(2)} k_{x} E_{z}^{2} M(0)$, where $\vec{e}_{\alpha}$ is the unit vector in the $\alpha$ direction, $\alpha=x, z$. Note that the corresponding crystallographic quadrupole component $\chi_{z z z z}^{(2)}$ is not excited because the $\vec{k}^{S P}$ vector has no $z$ projection. As such, the total second-order polarization $\vec{P}^{2 \omega}$ comprising both dipole and quadrupole contributions can be represented in the following way:

$$
\vec{P}^{2 \omega} \approx \chi_{z z z}^{(2, d)} E_{z}^{2} \vec{e}_{z}+\left(\chi_{x z z}^{(2, d)} E_{z}^{2} \vec{e}_{x}+i \chi_{z x z z}^{(2, Q)} k_{x} E_{z}^{2} \vec{e}_{z}\right) M(0)
$$

Here all the dipole terms except the first two are omitted, ${ }^{49}$ since the angle of incidence is relatively large and $E_{z}$ is enhanced due to the SPR in the most prominent way. The third nonlocal term is of quadrupole origin, whereas it is also odd with respect to the magnetization. This explains why the excitation of the SPR effectively changes the phase of the magnetic (odd-in magnetization) SH signal and not the nonmagnetic one, as seen in Fig. 3(b). The additional contribution, which only arises in the vicinity of the SPR, turns out to be magnetic due to the intrinsic symmetry of both the plasmon excitation and the quadrupole second-order susceptibility. The resonant nonlinear polarization $P^{(2 \omega, Q)}$ is directly proportional to the $k$ vector of a surface plasmon, which implies that the plasmon model is crucial for the explanation of the physics behind the observed results. Moreover, the phase of this additional 
contribution should be significantly different from that of the dipolar MSHG, and the phase shift $\varphi$ between magnetic and crystallographic MSHG contributions should experience a resonant change. However, although in a nondissipative medium there is a phase shift of $\pi / 2$ between electric dipolar and quadrupolar MSHG contributions, the observed phase shift stems from various SHG contributions, where not only phases but also their relative magnitudes play an important role.

\section{CONCLUSIONS}

To summarize, we demonstrated that an excitation of a surface plasmon at an interface of a ferromagnetic medium can not only enhance the SHG output, but also activate a quadrupole nonlinear-optical mechanism. This resonant SHG contribution is essentially nonlocal, and together with the nonlinear susceptibility symmetry it makes the SH field odd with respect to the sample magnetization. This results in substantial changes of the MSHG magnetic contrast and drastically modifies the phase of the nonlinear magneto-optical response.

\section{ACKNOWLEDGMENTS}

We would like to thank A. van Rooij, T. Toonen, and A. van Etteger for technical support. The authors acknowledge the support of The Netherlands Organization for Scientific Research (NWO), the 7th Framework Program (EU-FP7) IFOX Project, the European Research Council (ERC), and the Swedish Research Council (VR). *i.razdolski@ science.ru.nl

${ }^{\dagger}$ Present address: Technische Universität Kaiserslautern, ErwinSchrödinger-Straße 56, 67663 Kaiserslautern, Germany.

${ }^{1}$ J. A. Schuller, E. S. Barnard, W. Cai, Y. C. Jun, J. S. White, and M. L. Brongersma, Nat. Mater. 9, 193 (2010).

${ }^{2}$ W. L. Barnes, A. Dereux, and T. W. Ebbesen, Nature (London) 98, 824 (2003).

${ }^{3}$ J. M. McMahon, J. Henzie, T. W. Odom, G. C. Schatz, and S. K. Gray, Opt. Express 15, 18119 (2007).

${ }^{4}$ A. V. Kabashin, P. Evans, S. Pastkovsky, W. Hendren, G. A. Wurtz, R. Atkinson, R. Pollard, V. A. Podolskiy, and A. V. Zayats, Nat. Mater. 8, 867 (2009).

${ }^{5}$ H. P. Paudel and M. N. Leuenberger, Nano Lett. 12, 2690 (2012).

${ }^{6}$ B. Sepúlveda, A. Calle, L. M. Lechuga, and G. Armelles, Opt. Lett. 31, 1085 (2006).

${ }^{7}$ A. V. Zayats, I. I. Smolyaninov, and A. A. Maradudin, Phys. Rep. 408, 131 (2005).

${ }^{8}$ P. Nagpal, N. C. Lindquist, S.-H. Oh, and D. J. Norris, Science 325, 594 (2009).

${ }^{9}$ T. W. Ebbesen, H. J. Lezec, H. F. Ghaemi, T. Thio, and P. A. Wolff, Nature 391, 667 (1998).

${ }^{10}$ J. Beermann, T. Søndergaard, S. M. Novikov, S. I. Bozhevolnyi, E. Devaux, and T. W. Ebbesen, New J. Phys. 13, 3112 (2011).

${ }^{11}$ V. I. Belotelov, L. L. Doskolovich, and A. K. Zvezdin, Phys. Rev. Lett. 98, 077401 (2007).

${ }^{12}$ V. Belotelov and A. Zvezdin, J. Magn. Magn. Mater. 300, e260 (2006).

${ }^{13}$ S. Nie and S. R. Emory, Science 275, 1102 (1997).

${ }^{14}$ J. C. Quail and H. J. Simon, Phys. Rev. B 31, 4900 (1985).

${ }^{15}$ T. V. Murzina, I. A. Kolmychek, and A. I. Maydykovskiy, Bul. Russian Acad. Sci.: Phy. 76, 174 (2012).

${ }^{16}$ M. Kauranen and A. V. Zayats, Nat. Photonics 6, 737 (2012).

${ }^{17}$ B.Koene, M. Savoini, A. V. Kimel, A. Kirilyuk, and T. Rasing, Appl. Phys. Lett. 101, 013115 (2012).

${ }^{18}$ H. Feil and C. Haas, Phys. Rev. Lett. 58, 65 (1987).

${ }^{19}$ T. Katayama, Y. Suzuki, H. Awano, Y. Nishihara, and N. Koshizuka, Phys. Rev. Lett. 60, 1426 (1988).

${ }^{20}$ P. Fumagalli, C. Spaeth, and U. Rudiger, IEEE Trans. Magn. 31, 3319 (1995).

${ }^{21}$ A. V. Chetvertukhin, A. A. Grunin, A. V. Baryshev, T. V. Dolgova, H. Uchida, M. Inoue, and A. A. Fedyanin, J. Magn. Magn. Mater. 324, 3516 (2012).
${ }^{22}$ E. Melander, E. Ostman, J. Keller, J. Schmidt, E. T. Papaioannou, V. Kapaklis, U. B. Arnalds, B. Caballero, A. Garcia-Martin, J. C. Cuevas, and B. Hjörvarsson, Appl. Phys. Lett. 101, 063107 (2010).

${ }^{23}$ V. I. Safarov, V. A. Kosobukin, C. Hermann, G. Lampel, J. Peretti, and C. Marlière, Phys. Rev. Lett. 73, 3584 (1994).

${ }^{24}$ M. Born and E. Wolf, Principles Of Optics, 4th ed. (Pergamon, Oxford, 1970).

${ }^{25}$ Y. Shen, The Principles Of Nonlinear Optics (John Wiley \& Sons, New York, 1984).

${ }^{26} \mathrm{~A}$. Kirilyuk and T. Rasing, in Handbook of Magnetism and Advanced Magnetic Materials, Vol. 3: Novel Techniques for Characterizing and Preparing Samples, edited by H. Kronmüller and S. Parkin (John Wiley \& Sons, New York, 2007).

${ }^{27}$ R.-P. Pan, H. D. Wei, and Y. R. Shen, Phys. Rev. B 39, 1229 (1989).

${ }^{28}$ G. Tessier, C. Malouin, P. Georges, A. Brun, D. Renard, V. V. Pavlov, P. Meyer, J. Ferre, and P. Beauvillain, Appl. Phys. B 68, 545 (1999).

${ }^{29}$ H. A. Wierenga, M. W. J. Prins, D. L. Abraham, and Th. Rasing, Phys. Rev. B 50, 1282 (1994).

${ }^{30}$ M. Straub, R. Vollmer, and J. Kirschner, Phys. Rev. Lett. 77, 743 (1996).

${ }^{31}$ U. Conrad, J. Güdde, V. Jähnke, and E. Matthias, Phys. Rev. B 63, 144417 (2001).

${ }^{32}$ E. T. Papaioannou, V. Kapaklis, E. Melander, B. Hjorvarsson, S. D. Pappas, P. Patoka, M. Giersig, P. Fumagalli, A. Garcia-Martin, and G. Ctistis, Opt. Express 19, 23867 (2011).

${ }^{33}$ E. T. Papaioannou, V. Kapaklis, P. Patoka, M. Giersig, P. Fumagalli, A. Garcia-Martin, E. Ferreiro-Vila, and G. Ctistis, Phys. Rev. B 81, 054424 (2010).

${ }^{34}$ G. Ctistis, E. Papaioannou, P. Patoka, J. Gutek, P. Fumagalli, and M. Giersig, Nano Lett. 9, 1 (2009).

${ }^{35}$ H. Raether, Surface Plasmons (Springer-Verlag, Berlin, 1988).

${ }^{36}$ R. K. Chang, J. Ducuing, and N. Bloembergen, Phys. Rev. Lett. 15, 6 (1965).

${ }^{37}$ E. Schwarzberg, G. Berkovic, and G. Marowsky, Appl. Phys. A 59, 631 (1994).

${ }^{38}$ K. Kenmitz, K. Bhattacharyya, J. Hicks, G. Pinto, K. Eisenthal, and T. Heinz, Chem. Phys. Lett. 131, 285 (1986).

${ }^{39}$ R. Stolle, M. Loddoch, G. Marowsky, G. Berkovic, F. H. Kreuzer, and H. Leigeber, Langmuir 11, 3251 (1995).

${ }^{40}$ B. Koopmans, A. Janner, H. Wierenga, G. Sawatzky, and F. van der Woude, Appl. Phys. A 60, 103 (1995). 
${ }^{41}$ V. E. Babicheva and Y. E. Lozovik, Phys. Solid State 53, 804 (2011).

${ }^{42}$ S. Kujala, B. K. Canfield, M. Kauranen, Y. Svirko, and J. Turunen, Phys. Rev. Lett. 98, 167403 (2007).

${ }^{43}$ L. Cao, N. C. Panoiu, and R. M. Osgood, Jr., Phys. Rev. B 75, 205401 (2007).

${ }^{44}$ K. Sato, A. Kodama, M. Miyamoto, A. V. Petukhov, K. Takanashi, S. Mitani, H. Fujimori, A. Kirilyuk, and T. Rasing, Phys. Rev. B 64, 184427 (2001).

${ }^{45}$ V. Yannopapas, J. Phys.: Condens. Matter 20, 325211 (2008).
${ }^{46}$ F. X. Wang, F. J. Rodríguez, W. M. Albers, and M. Kauranen, New J. Phys. 12, 063009 (2010).

${ }^{47}$ V. V. Pavlov, G. Tessier, C. Malouin, P. Georges, A. Brun, D. Renard, P. Meyer, J. Ferre, and P. Beauvillain, Appl. Phys. Lett. 75, 190 (1999).

${ }^{48}$ V. V. Pavlov, J. Ferre, P. Meyer, G. Tessier, P. Georges, A. Brun, P. Beauvillain, and V. Mathet, J. Phys.: Condens. Matter 13, 9867 (2001).

${ }^{49}$ K.-H. Bennemann, Nonlinear Optics in Metals (Oxford University Press, Oxford, 1998). 